Images dans le monde ibérique et ibéricoaméricain

$8 \mid 2015$

L'Histoire et ses récits entre images, fictions et paratextes

\title{
La Malinche et les miroitements d'une image engloutie dans Le labyrinthe de la solitude
}

Monique Plâa

\author{
(2) OpenEdition \\ Journals \\ Édition électronique \\ URL : http://journals.openedition.org/agedor/317 \\ DOI : 10.4000/agedor.317 \\ ISSN : 2104-3353 \\ Éditeur \\ Laboratoire LISAA
}

Référence électronique

Monique Plâa, «La Malinche et les miroitements d'une image engloutie dans Le labyrinthe de la solitude », L'Âge d'or [En ligne], 8 | 2015, mis en ligne le 01 février 2016, consulté le 19 avril 2019. URL : http:// journals.openedition.org/agedor/317 ; DOI : 10.4000/agedor.317 


\author{
Monique PLÂA \\ U-PEM, LISAA, EA 4120
}

\title{
La Malinche et les miroitements d'une image engloutie dans Le labyrinthe de la solitude
}

\begin{abstract}
Résumé : Dans son essai, Octavio Paz, le poète, a recours à la poésie et à la création d'images. La Malinche est une image particulière : créée dans le chapitre IV du Labyrinthe où elle incarne la femme passive et violée des origines, elle est aussitôt engloutie. L'examen de quelques représentations iconographiques antérieures et contemporaines de la Malinche révèle que l'image sombre et tragique dont Paz semble faire l'emblème du mal-être mexicain, reflet d'une image créée par Orozco, est essentiellement une création personnelle. Mais pourquoi alors avoir définitivement englouti une image si puissante ? On peut émettre trois hypothèses : l'arbitraire qui a présidé à sa naissance, la centralité que cette image donnait à la Conquête alors que Paz entendait n'en faire qu'une étape parmi d'autres; la nécessité de déplacer le centre de gravité de l'analyse de l'être mexicain aux circonstances de l'être universel. Créée pour servir la pensée, l'image a disparu quand elle ne la servait plus.
\end{abstract}

Mots-clés : Le labyrinthe de la solitude - Malinche - Image - Peinture - Représentation

Resumen: El ensayista Paz es también poeta y su pensamiento es inseparable de las imágenes que lo organizan. La Malinche, creada en el capítulo IV del Laberinto, es la imagen de la mujer pasiva y violada de los orígenes. Ahora bien apenas creada esa formidable imagen desaparece del ensayo y de la obra de Paz. El examen de las representaciones anteriores y contemporáneas de la Malinche revelan que La Malinche del ensayo es esencialmente une creación personal. Tres hipótesis podrían explicar su asombrosa desaparición: su nacimiento arbitrario ; la excesiva importancia que la imagen daba a la Conquista ; la necesidad de desplazar el centro de gravedad del análisis del origen del ser mexicano a las circunstancias del ser universal. Creada para servir el pensamiento, la imagen de la Malinche desapareció cuando su creador reorientó su pensamiento.

Palabras clave: El laberinto de la soledad - Malinche - Imagen - Pintura - Representación

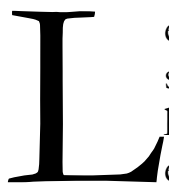

e miroir, le masque et le labyrinthe, qui déjà figure dans le titre de l'essai de Paz, sont quelques-unes de ces métaphores devenues images sur lesquelles se fonde l'écriture de Paz dans Le labyrinthe de la solitude. Mais, alors que le miroir, le masque et le labyrinthe ont une dimension métaphorique avant même que Paz n'en fasse des images structurantes de son essai, La Malinche est un cas tout à fait à part. Avant de figurer dans le chapitre IV du Labyrinthe de la solitude, on la trouve, d'abord, dans les chroniques de la Conquête et, ensuite, dans les livres d'histoire où elle n'est qu'un personnage secondaire; pourtant son statut si particulier de première alliée indienne du premier conquérant espagnol donne à La Malinche une charge symbolique dont témoignent déjà les représentations qu'ont laissées d'elle les peintres pour lesquels l'histoire est, en premier lieu, une source d'images.

La Malinche a d'abord été vendue par ses parents - apparemment par sa mère -, à un marchand maya du Yucatán qui, ensuite, l'a offerte comme butin à Cortés dans un lot de vingt femmes esclaves. Dans un premier temps, Cortés attribue La Malinche à Portocarrero, un capitaine auquel, dit-on, il réservait habituellement les femmes les plus belles. Quoique Cortés 
dans les "Cartas de relaciones » ne dise quasiment rien de l'arrivée de cette femme ni du rôle qu'elle jouera par la suite dans sa vie, les chroniques écrites par les contemporains de la Conquête la présentent comme un atout considérable dans le jeu du Conquérant dont elle sera la traductrice, l'ambassadrice et, très probablement, une conseillère avisée mais également son amante et, de surcroît, la mère d'un fils que Cortés fera légitimer par le pape avant de demander que lui soit attribué un habit de Santiago ; enfin, avant même d'organiser, pour son compte, un mariage de raison afin d'assurer sa promotion sociale en Espagne, Cortés se chargera d'établir socialement et financièrement sa compagne qu'il mariera finalement à l'un de ses capitaine préférés ${ }^{1}$.

Mais cette vie hors du commun, établie à partir de références éparses dans diverses chroniques, ne suffit surement pas à expliquer l'importance de la Malinche cinq cents ans après la Conquête. Si son nom, Malinche, figure de nos jours dans le titre de plusieurs romans et si on organise encore autour d'elle des colloques aussi importants que celui de Berlin en 2000 ou celui de Mexico qui, en 2001, réunit des chercheurs et des écrivains de première importance, ce n'est probablement pas la réalité de son histoire - dont on sait finalement peu de choses - qui peut l'expliquer et ce n'est probablement pas davantage le surgissement, au début du XIX , du terme " malinchisme » pour désigner une fascination excessive des élites mexicaines pour les étrangers ; sa célébrité, Malinche, la doit surtout au Labyrinthe de la solitude. C'est dans le chapitre IV de ce recueil que Paz a fabriqué une image de la Malinche dont la force et les enjeux se sont durablement inscrits dans l'imaginaire des lecteurs, et par-delà, dans l'imaginaire contemporain.

La Malinche est une de ces créatures qui nées sous la plume d'un écrivain vivent finalement leur vie dans la légende et le mythe. Pourtant, et ce n'est pas la moindre des surprises que réserve la lecture du Labyrinthe de la solitude, cette formidable image créée dans le chapitre IV disparaît dès le chapitre V. Dans la présentation qui suit, on se propose d'interroger les raisons de ce magistral engloutissement qui passe généralement inaperçu tant est permanente et toujours aussi vivante la fulgurante image de la Malinche créée par Paz. Dans un premier temps, on interrogera les raisons de la création de l'image de la Malinche dans le Labyrinthe de la solitude et ensuite on tentera d'apprécier la nature de l'image créée par Paz en la comparant très schématiquement à la représentation de la Malinche héritée des chroniques du XVII ${ }^{\mathrm{e}}$ siècle, des gravures du $\mathrm{XIX}^{\mathrm{e}}$ siècle et des peintures du $\mathrm{XX}^{\mathrm{e}}$ siècle. Dans un deuxième temps, on se risquera à émettre quelques hypothèses susceptibles de rendre compte du fatal engloutissement réalisé par son créateur même d'une image pourtant si brillamment créée.

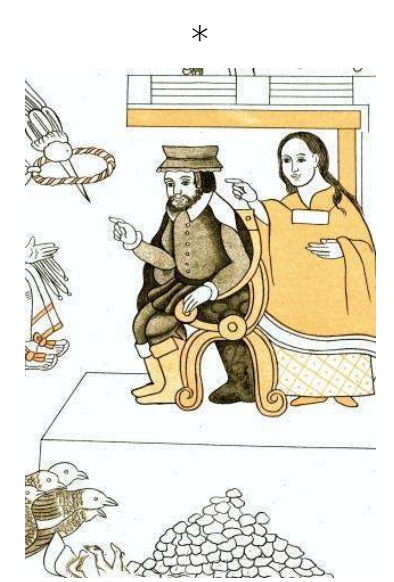

Cortés et la Malinche, Fragment du Lienzo de Tlaxcala, «Tenochtitlán », Dessins manuscrits, 1550, Musée National d'Anthropologie, Mexico

\footnotetext{
${ }^{1}$ Ces informations figurent dans les actes du colloque organisé par Margo Glantz en 2001: GLANTZ, Margo, (coordinadora), La Malinche, sus padres y sus hijos, Taurus, 2001.
} 


\section{La naissance de la Malinche dans l'organisation de l'essai}

La construction du Labyrinthe de la solitude est révélatrice de la manière dont Paz conçoit l'histoire dès les années $50^{2}$ : plus que l'observation du visible, l'Histoire c'est la mise en évidence de l'invisible. La démarche emprunte, entre autres, à la psychanalyse, et vise, à partir d'une fine observation de la situation présente, à explorer l'origine des conflits pour tenter de les comprendre afin de les résoudre.

Les derniers chapitres du Labyrinthe postulent que l'être mexicain mais, à vrai dire, l'homme en général, après avoir pris conscience de la nature de son mal-être pourrait enfin trouver sa place dans le monde. Entre les trois premiers chapitres de l'essai consacrés à l'observation du présent et les trois derniers qui envisagent les possibles solutions pour construire le futur, il y a 2 chapitres, les chapitres VI et VII, où Paz cherche dans l'Histoire du pays, celle qui va de la Conquête à la Révolution, à comprendre ce mal être dont les symptômes sont observables dans l'être mexicain contemporain. Le chapitre IV, qui permet de passer de l'observation synchronique des symptômes (chapitres I, II et III) à l'observation diachronique de leur manifestation dans l'histoire (chapitres V et VI), joue un rôle essentiel d'articulation puisque Paz entreprend, pour le lecteur, de rendre naturelle l'observation du passé à partir du présent.

L'élément à partir duquel l'observation du passé s'articule à celle du présent, c'est le langage et plus exactement le Cri poussé par tous les Mexicains le 15 septembre, jour qui commémore l'Indépendance du pays. Et c'est dans ce Cri, «Viva México, hijos de la chingada », que nait, dans la logique de l'essai de Paz, l'image de la Malinche. À partir de l'analyse des significations multiples et tout spécialement des connotations sexuelles du verbe "chingar», ce qui n'est pas sans rappeler le soin extrême que Freud portait au sens et au son des mots, Paz établit un rapport homme-femme particulièrement bien fait pour marquer durablement l'imagination du lecteur :

Las mujeres son seres inferiores porque, al entregarse, se abren. Su inferioridad es constitucional y radica en su sexo, en su "rajada", herida que jamás cicatriza. [...] La Chingada es la madre que ha sufrido metafórica o realmente, la acción corrosiva e infamante implícita en el verbo que le da nombre [...] El chingón es el macho, el que abre. La chingada, la hembra, la pasividad pura, inerme ante lo exterior (...) poder cínico del primero y la impotencia de la otra. La idea de violación rige oscuramente todos los significados. [...] para el español, la deshonra consiste en ser hijo de una mujer que voluntariamente se entrega, una prostituta ; para el mexicano es ser fruto de una violación [...] En suma, la cuestión del origen es el centro secreto de nuestra ansiedad y angustia ${ }^{3}$.

Si la psychanalyse cherche la source du traumatisme dans l'enfance, Paz la situe dans la conception même: "El hijo de la Chingada es el engendro de la violación, del rapto o de la burla"; "lo característico del mexicano reside, a mi juicio, en la violenta, sarcástica humillación de la Madre y en la no menos afirmación del Padre." Ainsi donc, l'origine du traumatisme se confond avec l'Origine en soi. Mais il y a plus : Paz confond l'Origine en soi avec l'Origine historique de la race métissée ; c'est, écrit Paz, qu'il n'est pas possible de ne pas voir dans le Père agresseur, l'étranger tout-puissant et, en particulier, le premier d'entre eux, Cortés - "Es imposible no advertir la semejanza que guarda la figura del macho con la del conquistador

\footnotetext{
2 En fait, avant d'articuler la solitude et l'histoire, Paz explore la question de la solitude en lien essentiel avec la poésie. Dès 1943, dans un essai intitulé « Poesía de soledad y poesía de comunión. », Paz écrit : « El poeta parte de la soledad, movido por el deseo, hacia la comunión. Siempre intenta comulgar, unirse, 'reunirse', mejor dicho, con su objeto: su propia alma, la amada, Dios, la naturaleza.» et Anthony Stanton de préciser : «Se desprende de este acercamiento entre poesía y religión que la meta es «tornar sagrado el mundo », STANTON, Anthony, Inventores de tradición : ensayos sobre poesía mexicana moderna, FCE, 1998, p. 182.

3 PAZ, Octavio, El laberinto de la soledad, Cátedra, 2010, pp. 212-217. Toutes les références à l'ouvrage renvoient à cette édition.

${ }^{4}$ Ibid., p. 217.
} 
español." ${ }_{5}$ De même, ajoute-t-il, il est impossible de ne pas voir dans la Mère violée, l'amante indienne de Cortés, la Malinche :

Si la Chingada es una representación de la Madre violada, no me parece forzado asociarla a la Conquista, que fue también una violación. No solamente en el sentido histórico sino en la carne misma de las indias. El símbolo de la entrega es la Malinche, la amante de Cortés. ${ }^{6}$

Ainsi donc, par simple glissement, Paz passe de l'Origine absolue, avec un O majuscule, à l'origine historique, celle du peuple métis né de la Conquête et, ce faisant, il passe de la Chingada, mère indéfinie, à la Malinche, l'amante de Cortés.

Ponctuellement, Paz signale bien que la réalité historique de Cortés et de la Malinche ne coïncident pas tout à fait avec l'image du Père violeur et de la Mère violée puisque la Malinche se donne à Cortés mais, de toute évidence, ce qui, pour Paz, importe par-delà les écarts avec l'histoire, c'est l'adéquation de l'image de la Chingada devenue Malinche au schéma d'interprétation mis en place dès le début du Labyrinthe de la solitude.

En effet, dès les trois premiers chapitres de l'essai, le lecteur comprend que, selon la vision de Paz, l'être mexicain est un être masqué puisqu'un visage d'apparence se superpose au visage authentique; déguisé de la sorte, l'être ne peut jamais être lui-même, ni jamais se retrouver ni, donc, jamais retrouver l'autre. Dans ce cadre-là, la Malinche, image maudite de la Mère des origines, devient la clé de voûte explicative de ce profond malaise ontologique: puisque le Mexicain renie ses origines, il est condamné, immanquablement, à la solitude -"reniega de su origen y se adentra solo en la vida histórica?". Or, comme tout lecteur le remarque d'entrée, Le labyrinthe se veut une réflexion pour envisager une solution au problème de la solitude. Par le simple fait de coïncider avec la Mère maudite des origines, reniée et honnie, l'image de la Malinche joue un rôle clé et ce d'autant plus que, dès le premier chapitre, Paz, conçoit l'avenir, et c'est d'ailleurs une grande originalité de sa pensée, comme un réancrage au passé, comme un retour à la source. La pensée de Paz s'inscrit dans un schéma mythique : construire le futur, c'est retrouver les temps premiers, le temps originel du vrai. De l'analyse de la Malinche, cette terrible image des Origines ontologiques et historiques d'un peuple né de la Conquête, devrait donc dépendre la possibilité ou non d'envisager un être mexicain libéré de la solitude, la possibilité ou non de construire l'avenir.

\section{Une image miroitement d'une autre image}

Avant d'interroger pourquoi cette image si essentielle, si forte et si terrible, contre toute attente, est très vite engloutie, un rapide détour par ses antécédents et par ses avatars contemporains à l'écriture du Labyrinthe dira à quel point la Malinche dont Paz fait le portrait est essentiellement une création et une créature d'Octavio Paz.

En 2001, Margo Glantz', lors du colloque qu'elle organise sur la Malinche, reprend à son compte le portrait flatteur qu'il est possible d'en faire à partir des chroniques de la Conquête : en effet, écrit-elle, les Espagnols l'appellent « Doña» tandis que les Indiens, qui rajoutent à son nom la marque le respect «Tzin », parent cette femme d'un tel prestige qu'il appellent Cortés « el señor Malinche ». D'autres intervenants soulignent l'attention que Cortés a manifesté pour le fils qu'il a eu avec la Malinche ; après l'avoir fait reconnaitre, Cortés a demandé un habit de Saint Jacques

\footnotetext{
${ }^{5}$ Ibid., p. 220.

${ }^{6}$ Ibid., p. 224.

${ }^{7}$ Ibid., p. 225.

${ }^{8}$ GLANTZ, Margo, « La malinche, la lengua en la mano », La Malinche, sus padres y sus hijos, op. cit, p. 90-95.
} 
pour ce fils qu'il disait aimer autant que celui qu'il avait eu, plus tard, avec son épouse espagnole. Bref, il faut apparemment attendre les premiers temps de l'Indépendance pour que, selon l'historien Jorge Alberto Manrique, surgisse, pour la première fois, le terme "malinchista » qui stigmatise ceux qui se tournaient vers les étrangers', et les étrangers, en ce temps-là, c'était les Espagnols d'Espagne mais aussi et surtout les Nord-américains dont on s'employait à copier la Constitution $^{10}$. On comprend que cette défiance à l'égard des partisans de l'Étranger s'exacerbe lors de la guerre contre les États-Unis et au moment de l'Intervention française, cependant, toujours selon Manrique ${ }^{11}$, l'accusation de «malinchismo » n'est virulente, c'est-à-dire au bord de l'insulte, que dans les milieux populaires et, en effet, si l'on en croit les gravures de l'époque, la Malinche, ici néoclassique et majestueuse et là coquette et orientalisante ${ }^{12}$, semble aussi éloignée qu'il est possible de l'être de l'idée de passivité et de viol : elle parait manifestement plus livrée à l'admiration des spectateurs qu'à leur malédiction ${ }^{13}$.

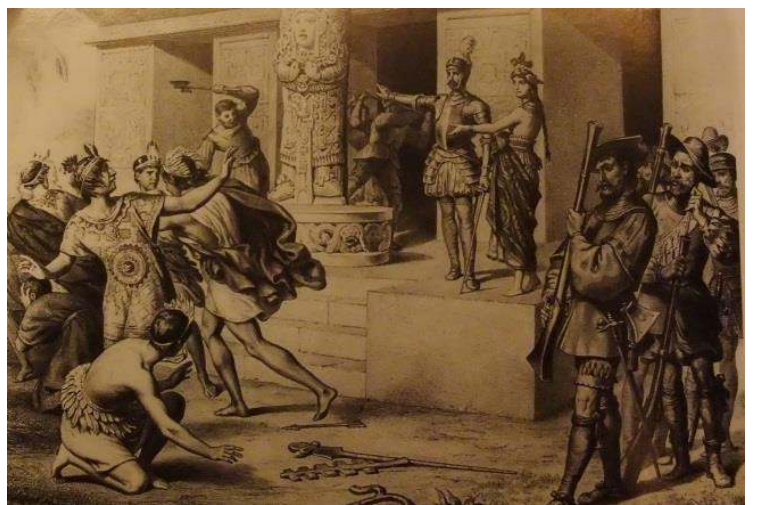

Hernán Cortés brise les idoles du temple de Vera Cruz au Mexique, Lithographie, XIX ${ }^{\mathrm{e}}$ s., BNF, Paris

Mais, en toute logique, c'est après la Révolution, quand la question de la nation par rapport aux puissances étrangères se pose à nouveau avec acuité exacerbant le nationalisme et, avec lui, le

\footnotetext{
${ }^{9}$ Selon Ramos, il y avait, au lendemain de l'Indépendance, un goût pour la culture étrangère qui résultait d'abord de la volonté de fuir la politique nationale : « (...) no se puede negar que el interés por la cultura extranjera ha tenido para muchos mexicanos el sentido de una fuga espiritual de su propia tierra. La cultura, en este caso, es un claustro en el que se refugian los hombres que desprecian la realidad patria para ignorarla ». Ramos voit dans le goût pour la culture étrangère la source du complexe d'infériorité qu'il analyse.

RAMOS, Samuel, « Perfil del hombre y la cultura en México » [1934], Obras completas, UNAM, 1990, p. 97.

${ }^{10} \mathrm{~S}$. Ramos tient le fait d'avoir adopté et adapté la Constitution des États-Unis pour le symptôme le plus manifeste de cette imitation de l'étranger qui, selon lui, est la marque d'un encombrant complexe d'infériorité -Ibid. p. 98.

11 «Surgió entonces el término malinchista, primero aplicado a los colaboradores de los extranjeros, luego a todos los que, en la idea popular, prefieren lo ajeno a lo propio. La Malinche se había convertido no sólo en la traidora sino (muy de acuerdo con la moral decimonónica y posterior) en la puta de Cortés. La asimilación traidora-puta conforma el personaje en la imaginería popular. En el mundo popular e informal la identificación Malinche-traidora-puta es una constante, pero no trasciende, sino esporádicamente a la cultura de mayores pretensiones. »

MANRIQUE, Jorge Enrique, « Malinche », in La malinche, sus padres y sus hijos, op. cit., p. 248.

${ }^{12}$ L'édition Cátedra n'est accompagnée que d'une seule image, sans titre ni date, p. 142. Cette somptueuse image de la Malinche et de Cortés en couple galant qui rappelle, en plein XIX ${ }^{\mathrm{e}}$ siècle, les jeux de l'amour de Mars et Vénus tels que les représentaient le XVII ${ }^{\mathrm{e}}$ siècle contredit de manière flagrante l'image créée par Octavio Paz. On ne sait qui a choisi cette image mais on aurait beaucoup aimé connaitre les arguments qui ont présidé à ce choix.

13 Dans la version du «Diccionario universal de historia y de geografía » revue par les Mexicains en 1854 - la première édition est espagnole et date 1847, il n'y a aucune définition de «Malinche » ni du «malinchismo »; en revanche, il y a un portrait très flatteur de Marina à l'article «Conquistadores »!; dans le tome 2 du Dictionnaire, on ne trouve à la rubrique "Nueva España », assez longue et soigneusement écrite, aucune mention de la Malinche. Le discours officiel semble ici ne retenir que la belle image de la Malinche. Quant à Justo Sierra, en 1888, dans un livre édité à l'usage des enfants, il propose une lecture de l'histoire du Mexique organisée autour de la notion de progrès : l'origine et le métissage ne font l'objet d'aucune interrogation. SIERRA, Justo, Elementos de Historia general para las escuelas primarias, México, Imprenta de E. Dublan y comp., 1888.
} 
«malinchismo » que l'image de la Malinche aurait dû devenir un fort enjeu de la représentation de l'identité.

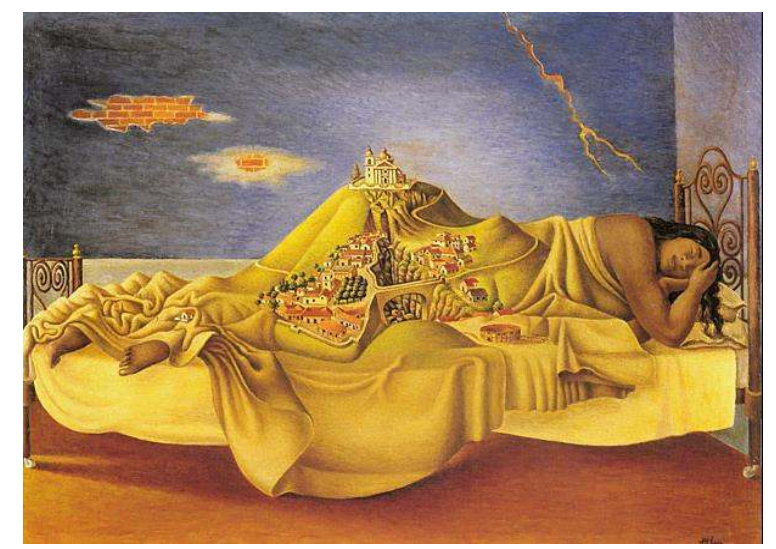

Antonio Ruiz, El sueño de la Malinche, 1939, huile sur bois, 30 x 40 cm, Galería de Arte Mexicano, Mexico

Or en 39, Antonio Ruiz peint un tout petit tableau qui représente une Malinche qui se confond avec le volcan qui porte son nom ; et sa pose allongée et alanguie fait d'elle, l'Indienne au visage paisible, une manière de déesse porteuse de la civilisation coloniale à venir, comme en atteste le paysage urbain qui forme son corps. Particulièrement attaché à la miniature, Antonio Ruiz a toujours porté un regard amusé et moqueur sur les amples fresques didactiques dont les muralistes ornaient les bâtiments publics. On pourrait donc imaginer que sa Malinche, apaisée et apaisante, est le contrepoint de quelque Malinche livrée à la vindicte du spectateur qui serait née, par exemple, sous le pinceau de Diego Rivera dont Octavio Paz n'a cessé de déplorer qu'il gâte son talent par un marxisme et un nationalisme aussi candides que tapageurs ${ }^{14}$.

Si Diego Rivera avait eu cette candeur nationaliste dont l'accuse Paz, à l'heure où il peint dans le Palais National une fresque qui relit l'histoire du Mexique à partir du peuple et pour le peuple, il aurait dû, en bonne logique, associer la Malinche au camp des forts et faire d'elle un de ces portraits, comme on en trouve plusieurs dans la fresque, où le trait forcé propre de la caricature facilite la compréhension critique.

14 Voir, PAZ, Octavio, «Los privilegios de la vista 2 », Obras completas, T. VII. Paz a consacré un volet important de l'histoire de l'art mexicain aux muralistes et, entre autres, à l'œuvre de Rivera. 


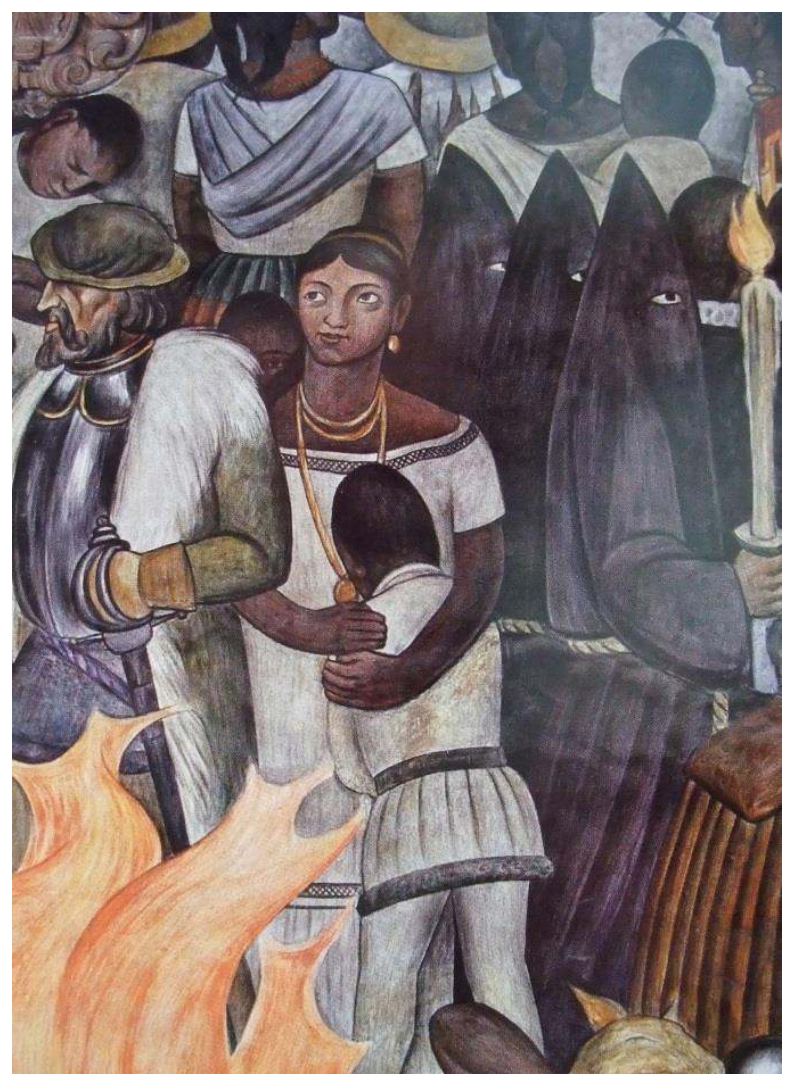

Diego Rivera, Histoire du Mexique, fresque, 1929-30, Palais National

Or, dans la fresque du Palais National, la Malinche, quoique dans un léger retrait, est présente et illuminée: le regard noir et vif, elle paraît bien plus près de l'image que donnent d'elle les chroniqueurs - Diaz del Castillo, en particulier- que de la femme, passivement violée, reniée et insultée d'Octavio Paz. Paz écrit que c'est dans une insulte qu'il a découvert le reniement de la mère des Origines et donc le reniement de la Malinche qui fonde le métissage mais, dans la fresque du Palais national qui, précisément parce qu'elle se trouve dans le lieu même du pouvoir, entend relire l'histoire au nom du peuple, la Malinche ne correspond pas du tout à ce que Paz prétend qu'elle est pour le peuple. 


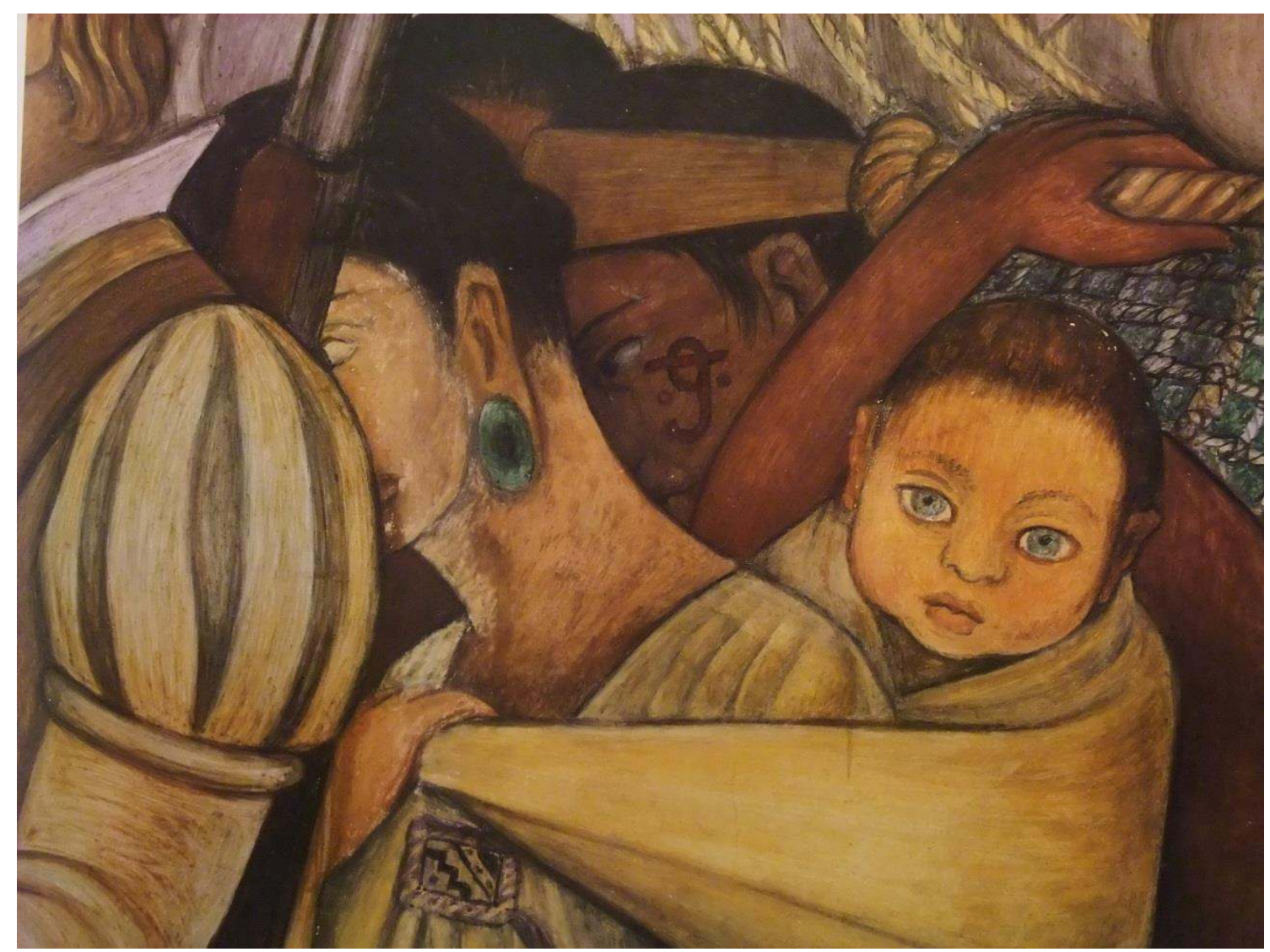

Diego Rivera, La conquête du Mexique, 1952, fresque, Palais National, Mexico.

En 1952, Rivera peint une nouvelle version de l'arrivée des Espagnols, il concentre dans cette nouvelle fresque de multiples scènes violentes où Cortés est, cette fois, sévèrement caricaturé, en revanche, quoique découpée entre l'homme blanc dont l'épaule et le fusil lui cachent à demi le visage et homme indien, sinistrement marqué comme butin de guerre, la femme qui figure la Malinche n'est ni caricaturée, ni dégradée et, qui plus est, elle oriente le regard du spectateur vers l'enfant qu'elle porte sur son dos. Cet enfant, spectaculaire par la plaisante douceur de ses traits et surtout par l'immensité de ses yeux bleus, semble récuser frontalement la lecture de Paz : Rivera crée une image gracieuse et légère, une image amusée et amusante aussi distante qu'il est possible de l'être de l'image tragique des origines dont le texte de Paz dit avoir descellé la terrible présence dans le Cri «Viva México, hijos de la chingada».

Bref, à ce stade, on peut se risquer à faire l'hypothèse que l'unique Malinche qui a à voir avec celle de Paz, c'est la Malinche d'Orozco que Paz, du reste, mentionne explicitement dans Le labyrinthe de la solitude - "Al repudiar a la Malinche - Eva Mexicana, según la representa José Clemente Orozco en su mural de la Escuela Nacional preparatoria- el mexicano rompe sus ligas con el pasado, reniega de su origen y se adentra solo en la vida histórica." ${ }^{\text {,15 }}$

15 PAZ, Octavio, El laberinto de la soledad, op. cit., p. 225. 


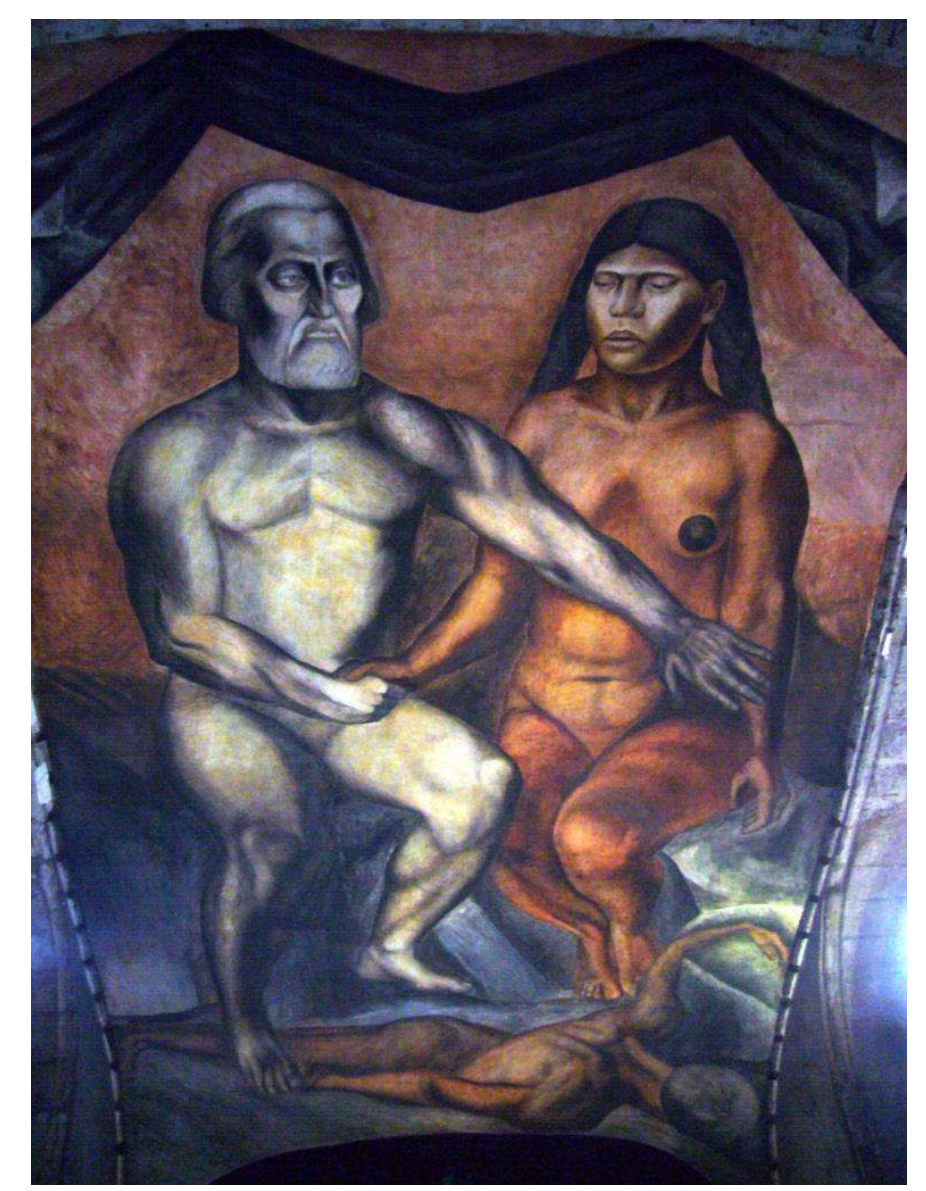

José Clemente Orozo, Fresque, 1926, Colegio San Ildefonso, Mexico.

Une analyse un peu rigoureuse mettrait vite en évidence que la Malinche du Labyrinthe de la solitude n'est pas exactement celle d'Orozco mais, ici, on émettra simplement l'hypothèse que la Malinche de Paz pourrait bien être née moins de l'analyse archéologique d'une insulte révélatrice des tréfonds de l'identité mexicaine que du miroitement d'une autre image, et si tel est le cas, si la Malinche de Paz est essentiellement une création personnelle, c'est donc que Paz y tient tout particulièrement comme semble, du reste, l'indiquer le rôle que joue cette image dans la structure du livre.

Or, sous réserve de vérifications plus méticuleuses, force est de constater que, aussitôt née, la Malinche disparait complétement et définitivement de l'univers de Paz.

En 1977, alors qu’il évoque le Cri du 15 septembre, Paz écrit :

Se nos escapará el sentido profundo del ritual del 15 de setiembre, el Grito, si no advertimos que es una ceremonia dual: por una parte, es una resurrección simbólica de la nación mexicana y, por la otra, una consagración de la autoridad legítima. Más que una celebración democrática es una liturgia política impregnada de religiosidad. ${ }^{16}$

Dans le Labyrinthe, le Cri du 15 septembre est le point de départ d'une exploration au terme de laquelle Paz dévoile, pour son lecteur, l’image de la Malinche. En 77, le Cri est envisagé d'une toute autre manière et, dans la nouvelle analyse, il n'y a plus trace de la Malinche. On pourrait alors croire que la Malinche a disparu de l'œuvre de Paz au cours du temps, dix ou vingt ans après sa création, or, précisément, non: pour la plus grande surprise du lecteur, il s'avère que cette formidable image de la Mère reniée et honnie crée par Paz dans le chapitre IV a été engloutie dès le chapitre $\mathrm{V}$ du Labyrinthe de la solitude. On ne peut donc que se demander pourquoi cette

\footnotetext{
16 PAZ, Octavio, « Xavier Villaurutia en persona y su obra [1977] », Obras completas, IV, 1994, p. 260.
} 
stupéfiante et formidable Malinche, à peine créée, a été aussitôt engloutie corps et biens par Paz lui-même. C'est à cette question que nous allons maintenant tenter de répondre.

\section{Hypothèses pour un engloutissement}

La première explication possible pour rendre compte de sa disparition est peut-être à chercher dans la naissance même de la Malinche : en effet, il s'avère que La Malinche de Paz est née d'une association pour le moins libre entre la Chingada, une figure indéfinie au contenu virulent et diversement injurieux, et la Malinche, la femme indienne historiquement associée à la Conquête. Sur ce recoupement audacieux et arbitraire, Paz a lancé une analyse sans doute un peu forcée et sans doute, pour cette raison même, quelque peu porteuse de sa propre fragilité. En 2001, lors du colloque organisé par Margo Glantz, à propos de la Malinche ouverte, violée, image de l'indienne soumise au père européen qui expliquerait une faille existentielle du Mexicain contemporain, l'historien Manrique, avec doigté, signale qu'il faudrait sans doute remettre en question la fabrication de la Malinche par Paz :

Octavio Paz ha escrito brillantes páginas donde la Malinche se relaciona con la violada, la "rajada", la imagen de una madre india sometida violentamente por el padre europeo, que pesa sobre la debilidad existencial de los mexicanos. Hipótesis que en su momento resultó reveladora, escandalosa y contundente, y que quizá ahora haya que tomar con más cuidado. ${ }^{17}$

Avec moins d'élégance mais plus d'humour, lors de ce même colloque, l'essayiste Carlos Monsiváis déclarait que la Malinche n'a de fait rien avoir avec Eve ou la Mère avec Majuscule et d'ajouter que la Chingada, déjà du temps du Labyrinthe, n'était qu'un mot haut en couleur mais vide de sens : "la Chingada para estas fechas es sólo un recurso del vocabulario pintoresquista"18. Si donc l'image de la Malinche naît d'une trop libre association de mots et d'une interprétation un brin "sauvage ", on pourrait croire que cela a suffi pour que Paz se détourne aussitôt de son formidable colosse aux pieds d'argile. Mais il est fort probable qu'il y a bien d'autres hypothèses possibles pour expliquer cet engloutissement complet et définitif, on se contentera, ici, d'en signaler deux.

Comme il a été dit, dans le chapitre IV, la Malinche est le point d'articulation entre, d'une part, l'observation des symptômes révélateurs d'un traumatisme chez les contemporains et, d'autre part, l'exploration des manifestations du traumatisme dans l'Histoire ; dans cette optiquelà, la Malinche faisait immanquablement de la Conquête le repère essentiel entre tous : celui de la fondation première et conflictuelle du Mexique; or, précisément, Le labyrinthe de la solitude contredit et contrarie la tentation de faire de la Conquête le centre de gravité de l'Histoire du Mexique. Paz, dès Le labyrinthe - et constamment par la suite- amoindrit l'importance de la Conquête : d'abord, il signale que les Aztèques ont été défaits moins par les Espagnols que par leurs propres dieux qui les ont abandonnés; ensuite, Paz ne cessera de réaffirmer que les Espagnols, loin d'anéantir le pouvoir aztèque, de fait, en prolongent l'organisation pyramidale ce qui implique que la Conquête ne saurait être tenue pour une rupture absolue -, et, enfin et surtout, Paz va faire du premier christianisme de la Colonie un temps de référence, un temps en grande partie exemplaire et, sans doute une des explications les plus plausibles de l'engloutissement de l'image de la Malinche. En effet, très vite, dès le chapitre V, le christianisme des premiers temps de la Colonie représente l'ouverture universelle: "Forma y sustancia

\footnotetext{
${ }^{17}$ MANRIQUE, Jorge-Alberto, « Malinche », La Malinche, sus padres y sus hijos, Margo Glantz (coordinadora), op. cit., p. 248-249.

${ }^{18}$ MONSIVÁIS, Carlos, « La malinche y el malinchismo », La Malinche, sus padre y sus hijos, op. cit., p. 192.
} 
pactan $(\ldots)$. (...) todo se corresponde y unos mismos conceptos y una misma voluntad rigen los ánimos. El hombre, por más humilde que sea su condición, no está solo. Ni tampoco lo está la sociedad $»^{19}$.

Or c'est précisément à partir du concept d'ouverture universelle à l'autre qu'Octavio Paz fonde sa vision dialectique de l'Histoire dès le chapitre V. Ainsi donc, la Conquête, et avec elle la Malinche, se trouve éclipsée par les premiers temps de la Colonie, des temps exemplaires de la communion puisque, on vient de le lire, "l'homme n'est plus seul et la société non plus». Insensiblement, la Mère des Origines ce n'est plus la Malinche, - celle-là même qui était cause de reniement et de solitude -, mais la religion chrétienne - qui exorcise la solitude. Par conséquent, la dialectique de la solitude ne passe plus par la Malinche mais par Sor Juana Inés de la Cruz, la femme - non plus indienne mais espagnole- qui, voyant le christianisme se fermer, est la première à se cacher et donc, la première à porter le masque qui dit l'identité falsifiée de l'être mexicain ${ }^{20}$.

Désormais, et ce dès le chapitre $\mathrm{V}$, le schéma qui organise la pensée dans Le labyrinthe est définitivement en place. Toutes les étapes de l'Histoire seront envisagées à partir de l'opposition ouvert-fermé. Or la Malinche qui précisément n'est qu'ouverture ne pouvait que brouiller cette opposition : et c'est là, dans le risque certain d'un brouillage, que se trouve l'ultime possible explication de son engloutissement ${ }^{21}$. En effet, l'opposition ouvert-fermé permet à Paz d'examiner toutes les formes de pouvoir pour mieux rejeter les systèmes politiques qui briment la réalité de l'être : le problème n'est dès lors plus centré sur les fondations ontologiques de l'Être, comme l'induisait l'image de la Malinche en Mère des Origines reniée et honnie, mais déplacé sur le pouvoir, c'est-à-dire, sur les circonstances de l'Être. Alors que le problème ontologique était quasiment insoluble, la nouvelle manière de configurer la dialectique de la solitude rend la possibilité d'une résolution bien plus envisageable pour l'être mexicain dont Paz postule qu'il est en attente d'authenticité et de communion comme le révèlent les moments d'explosion que sont La Fête et la Révolution en particulier; en faisant l'examen des formes diverses que prend le pouvoir, Octavio Paz dénonce leur inadéquation à l'Être. La Malinche centrait le problème sur une tache de l'Origine, sur une manière de péché originel de l’Être mexicain, le schéma qui organise finalement la dialectique de la solitude centre le problème sur le pouvoir comme expression inadéquate à l'Être universel dont le salut ontologique peut dès lors être envisagé comme un retour aux origines, certes, mais non plus à celles de la Malinche, finalement engloutie, mais à celles qui fondent, selon Octavio Paz, l'être de tous les êtres : l'imagination, le rêve, le mythe, de l'érotisme, bref, selon la belle expression de Paz, la « pure vie ».

19 PAZ, Octavio, El laberinto de la soledad, op. cit., p. 313.

${ }^{20} \mathrm{Paz}$ consacrera un livre à Sor Juana, bien plus tard, en 1982. Il reprend alors en partie son premier cadre d'analyse, celui du Labyrinthe : «La palabra de Sor Juana se edifica frente a una prohibición ; esa prohibición se sustenta de una ortodoxia, encarnada en una burocracia de prelado y jueces. » À ce cadre, s'ajoute une perspective tout à fait nouvelle qui met le reniement de Sor Juana en relation avec celui des artistes et des intellectuels du $\mathrm{XX}^{\mathrm{e}}$ siècle : "En el siglo XX, por una suerte de regresión histórica, abundan también los ejemplos de escritores e ideólogos transformados en acusadores de sí mismos. La semejanza entre los años finales de sor Juana y estos casos contemporáneos me hicieron escoger como subtítulo de mi libro el de la sección última : Las trampas de la fe. » On remarquera, au passage, que la Malinche n'est même pas répertoriée dans l'index alphabétique de cet essai alors que Cortés y figure à sept reprises. PAZ, Octavio, «Sor Juana Inés de la Cruz o las trampas de la fe », Obras completas, T. V, FCE, 1994, p. 22.

${ }^{21}$ La Malinche, sous plus d'un angle, pouvait signifier l'ouverture à l'autre, cette ouverture que Paz a toujours recherchée. Paz, dans son autobiographie, fait de lui le portrait d'un enfant stigmatisé parce que tenu pour étranger, « un gringo, un franchute o un gachupín, les daba lo mismo. », PAZ, Octavio, Itinerario, Biblioteca de Bolsillo, 1994, p. 13. Par ailleurs, parmi les références privilégiées dans l'ensemble de l'œuvre de Paz, il y a les Contemporáneos; ils figurent déjà dans Le labyrinthe et plus de quarante ans plus tard, on les retrouve dans Itinerario. Paz dénonce toujours l'injustice qui leur a été faite : au lieu de célébrer leur ouverture, on l'a condamnée en les accusant d'être en dehors de la nation. La Malinche, par le parti-pris de regarder ailleurs, est très près de l'ouverture si chère à Paz. Maudite par le peuple (dans la lecture qu'avait choisi d'en faire Paz, s'entend) et finalement porteuse de valeurs proches de celles auxquelles Paz tient particulièrement, il est probable que La Malinche était susceptible de créer un effet de brouillage dont on peut supposer qu'il a contribué à la disparition de la remarquable image créée au chapitre IV du Labyrinthe de la solitude. 
La Malinche d'Octavio Paz a été pensée dans une démarche explicative sans doute largement inspirée de la psychanalyse qui voit dans l'image du Père et de la Mère la source de beaucoup de conflits. Cette Malinche, image forte et inoubliable crée par un poète qui réinterprète un personnage historique, semble pourtant, comme le suggère entre autres l'œuvre de Diego Rivera, assez éloignée de la manière dont les Mexicains se représentent l'alliée de Cortés. Née probablement d'une libre interprétation de la Malinche d'Orozco, celle d'Octavio Paz est une formidable image dont le lecteur comprend d'entrée qu'elle doit jouer un rôle essentiel dans la dialectique de la solitude. Or précisément, la manière de concevoir cette dialectique évolue ${ }^{22}$ et entraîne un déplacement du centre de gravité de l'œuvre: si la Mère de référence, c'est la Malinche, la Conquête devient l'objet prioritaire de toute réflexion sur l'Histoire. Or, Octavio Paz, qui célèbre l'ouverture à l'Autre et l'universalité, tient tout spécialement à faire de la Conquête une étape comme une autre et, en conséquence, le centre de gravité de sa lecture de l'Histoire se déplace sur la Colonie dont le catholicisme ouvert et universel de la première époque, en contraste avec le catholicisme fermé qui suivra, forme la matrice du schéma dialectique de la solitude: désormais recentré sur les formes du pouvoir comme autant de circonstances qui briment l'Être, le schéma dialectique demande à ce que l'image de la Malinche soit engloutie. Le labyrinthe de la solitude est le premier essai d'Octavio Paz, ambitieuse et magistrale sous plus d'un aspect, la lecture du Mexique que le livre propose n'est pas le résultat d'une pensée aboutie mais d'une pensée qui se cherche et qui, le cas échéant, se réoriente. Octavio Paz, avec quelque injustice d'ailleurs, reprochait à Cortés d'avoir abandonné la Malinche dès lors qu'elle ne lui était plus d'aucune utilité, le lecteur, en toute justice, pourrait reprocher à Octavio Paz d'avoir englouti sans hésiter la belle image de la Malinche dont il était le père dès lors qu'elle encombrait plus qu'elle ne servait la pensée en construction dans Le labyrinthe de la solitude. 